\title{
Suspected paclitaxel-induced pneumonitis
}

\author{
Kohei Shitara ${ }^{1}$, Eiji Ishi ${ }^{2}$, Makoto Kondo $^{2}$, and Yuh SaKata ${ }^{1}$ \\ ${ }^{1}$ Department of Medical Oncology, Misawa City Hospital, 4-1-10 Chyuo-cho, Misawa, Aomori 033-0001, Japan \\ ${ }^{2}$ Department of Gastroenterology, Kameda Medical Center, Kamogawa, Japan
}

\begin{abstract}
We report two patients with advanced gastric cancer with suspected paclitaxel-induced interstitial pneumonitis. Case 1, a 66-year-old man with recurrent gastric cancer, was treated weekly with paclitaxel. After 11 administrations of paclitaxel, he developed a nonproductive cough and dyspnea. Computed tomography (CT) scan showed extensive bilateral areas of ground-glass attenuation. He did not respond to corticosteroid, and died 57 days after the last paclitaxel administration. Case 2, a 61-year-old man with gastric cancer and liver metastasis, was treated with weekly paclitaxel. The liver metastasis was dramatically reduced in size, but he developed a nonproductive cough and dyspnea after 21 administrations of paclitaxel. He did not respond to high-dose corticosteroid, and died 50 days after the last paclitaxel administration. Although it is a rare complication, paclitaxel-induced interstitial pneumonitis needs to be considered in the differential diagnosis when patients treated with paclitaxel present with fever, nonproductive cough, and dyspnea.
\end{abstract}

Key words Paclitaxel · Interstitial pneumonitis · Gastric cancer

\section{Introduction}

Paclitaxel (PTX) (Taxol; Bristol-Myers Oncology, Princeton, NJ, USA) is a chemotherapeutic agent widely used in the management of a range of malignant disorders, including carcinoma of the lung, breast, and ovary, and gastric cancer. Common PTX toxicities include neutropenia, neuropathy, and hypersensitivity reactions [1]. Pulmonary toxicity related to the administration of PTX is a rare event, but can be fatal [2]. We

Offprint requests to: $\mathrm{K}$. Shitara

Received: March 30, 2006 / Accepted: June 5, 2006 describe two patients with advanced gastric cancer in whom interstitial pneumonitis developed after several courses of a chemotherapy regimen based on weekly PTX administration.

\section{Case report}

Case 1

A 66-year-old man with a ten-pack-a-day history of cigarette smoking was diagnosed with gastric cancer at Kameda Medical Center. He underwent distal gastrectomy with D2 dissection in December 2003 (final stage, T3N2M0 stage IIIB). S-1 was initiated as adjuvant chemotherapy, but it was discontinued due to rash, stomatitis, and diarrhea.

One year, he developed jaundice because of abdominal lymph node recurrence. A biliary stent was inserted and weekly PTX chemotherapy was begun after the jaundice resolved. PTX was given on days 1, 8, and 15, at a dose of $80 \mathrm{mg} / \mathrm{m}^{2}$, for $1 \mathrm{~h}$. Premedication with dexamethasone $(8 \mathrm{mg})$, ranitidine $(50 \mathrm{mg})$, and diphenhydramine $(50 \mathrm{mg})$ was administered prior to the administration of PTX. The chemotherapy courses were repeated every 3 weeks. A computed tomography (CT) scan before chemotherapy with PTX was started showed minimal consolidative changes and subpleural lines in the bilateral lower lung fields (Fig. 1A). After 8 administrations of PTX, a CT scan was performed to detect lung metastasis. Minimal consolidative areas were observed, and the subpleural lines showed slight exacerbation (Fig. 1B). A CT scan of the abdomen showed the size of the lymph node metastasis was stable. After 10 administrations of PTX, he developed a transient cough and a low-grade fever, which resolved spontaneously in 2 days. Four days after 11 administrations of PTX, he developed a nonproductive cough and progressive dyspnea. Physical examination revealed 


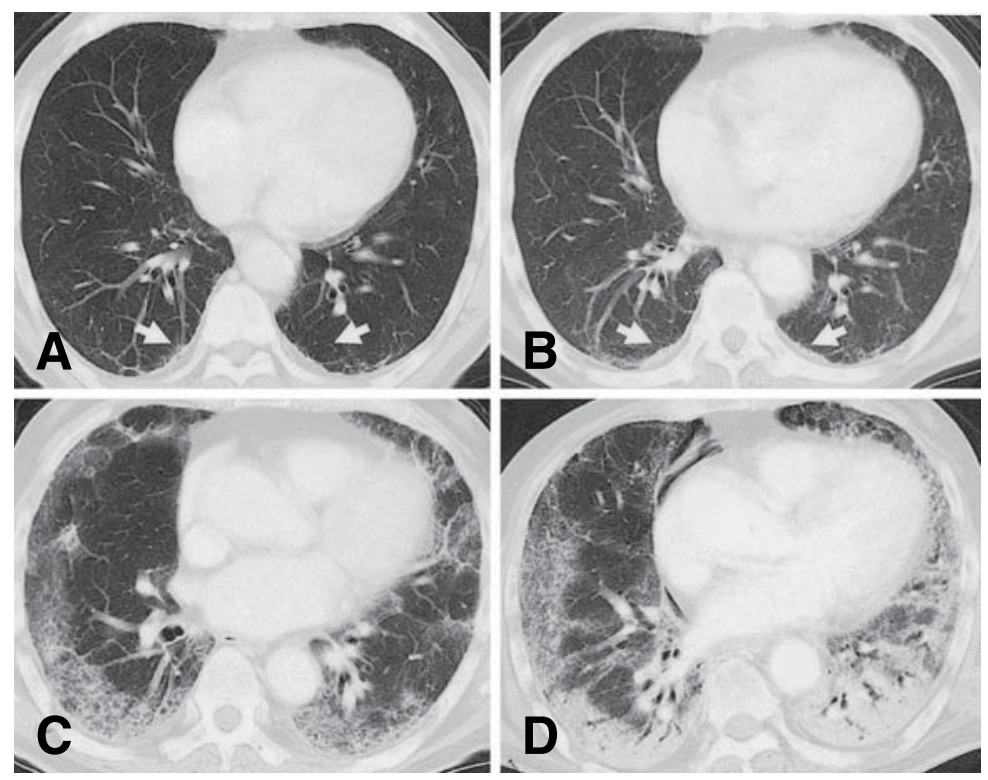

Fig. 1A-D. Computed tomography (CT) scans in case 1. A CT scan before paclitaxel (PTX) chemotherapy was started. It showed minimal consolidative changes (arrow) and subpleural lines (arrow) in the bilateral lower lung fields. B CT scan after eight administrations of PTX. Minimal consolidative areas were observed, and the subpleural lines showed slight exacerbation (arrows). C CT scan 4 days after 11 administrations of PTX, showing extensive bilateral areas of ground-glass attenuation, especially in peripheral regions. D CT scan 1 month after 11 administrations of PTX. It showed further exacerbated ground-glass attenuation and consolidative changes in bilateral lungs fine crackles in the bilateral lungs. Arterial blood gas analysis was as follows: $\mathrm{pH}, 7.49 ; \mathrm{PCO}_{2}, 31.2 \mathrm{mmHg}$; and $\mathrm{PO}_{2}, 45.4 \mathrm{mmHg}$. Laboratory values were as follows:

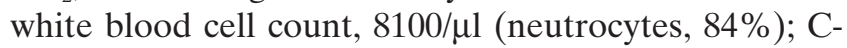
reactive protein, $10.4 \mathrm{mg} / \mathrm{dl}$; lactate dehydrogenase (LDH), $724 \mathrm{IU} / \mathrm{ml}$; and sialylated carbohydrate antigen KL-6 (KL-6), 2610 U/ml. A CT scan showed extensive bilateral areas of ground-glass attenuation, especially in peripheral regions (Fig. 1C). Cultures of sputum and blood were negative for infection. $\beta$-D-Glucan was within the normal range. Drug-induced lymphocyte stimulation test (DLST) for PTX was negative. Druginduced interstitial pneumonitis related to PTX was suspected on the basis of the clinical course and findings. High-dose steroid therapy (IV methylprednisolone $1 \mathrm{~g}$ daily for 3 days; IV prednisolone $50 \mathrm{mg}$ daily for the next 4 days) was given, but it was ineffective. He developed respiratory failure requiring tracheal intubation and mechanical ventilation, and a tracheotomy was performed. A total of four cycles of high-dose steroid and two cycles of cyclophosphamide were given, but all were ineffective. A CT scan obtained 1 month after the diagnosis of pneumonitis showed further exacerbated ground-glass attenuation and consolidative changes in the bilateral lungs (Fig. 1D). He died 57 days after the last PTX administration.

\section{Case 2}

A 61-year-old man with a history of myocardial infarction at the age of 50 years and a 20-pack-a-year history of cigarette smoking was diagnosed with gastric cancer at Kameda Medical Center in June 2004. A CT scan before chemotherapy was begun showed a $94 \times$ 80-mm metastatic tumor in the left lobe of the liver (Fig. 2A).

Combination therapy of S-1 and cisplatin was begun. $\mathrm{S}-1$ was given consecutively, at $80 \mathrm{mg} / \mathrm{m}^{2}$, on days 1 to 21. Cisplatin was given at $60 \mathrm{mg} / \mathrm{m}^{2}$ on day 8 . The treatment was repeated every 5 weeks. After two courses of this treatment, the liver metastasis remained stable (Fig. 2B), but the gastric tumor had become enlarged, so weekly PTX chemotherapy was started as second-line treatment. PTX was given at $80 \mathrm{mg} / \mathrm{m}^{2}$ on days 1,8 , and 15. This treatment was repeated every 3 weeks. Premedication was similar to that in case 1. A CT scan before PTX chemotherapy showed slight consolidative changes and subpleural lines (Fig. 3A), but the patient noticed no symptoms. After four administrations of PTX, the dose of paclitaxel was reduced to $70 \mathrm{mg} / \mathrm{m}^{2}$, due to the occurrence of grade 3 febrile neutropenia. After six administrations, the liver metastasis was reduced in size $(38 \mathrm{~mm} ; 59 \%$ reduction; Fig. 2C), and after 20 administrations, the liver metastasis was further reduced in size (Fig. 2D). Two days after 21 administrations of PTX, he was hospitalized with fever, nonproductive cough, and progressive dyspnea. A CT scan showed extensive bilateral areas of ground-glass attenuation with honeycomb changes (Fig. 3B,C). Arterial blood gas analysis was as follows: $\mathrm{pH}, 7.42 ; \mathrm{PCO}_{2}$, $35 \mathrm{mmHg}$; and $\mathrm{PO}_{2}, 62 \mathrm{mmHg}$. Laboratory values were as follows: white blood cell count, $4400 / \mu l$ (neutrocytes, $70 \%$ ); C-reactive protein, $8.85 \mathrm{mg} / \mathrm{dl}$; LDH, $942 \mathrm{IU} / \mathrm{ml}$; and KL-6, $4340 \mathrm{U} / \mathrm{ml}$. Cultures of sputum and blood were negative for infection. DLST for PTX was negative. High-dose steroid therapy, cyclophosphamide, and cyclosporine were given, but all were ineffective. He died 50 days after the last PTX administration. 

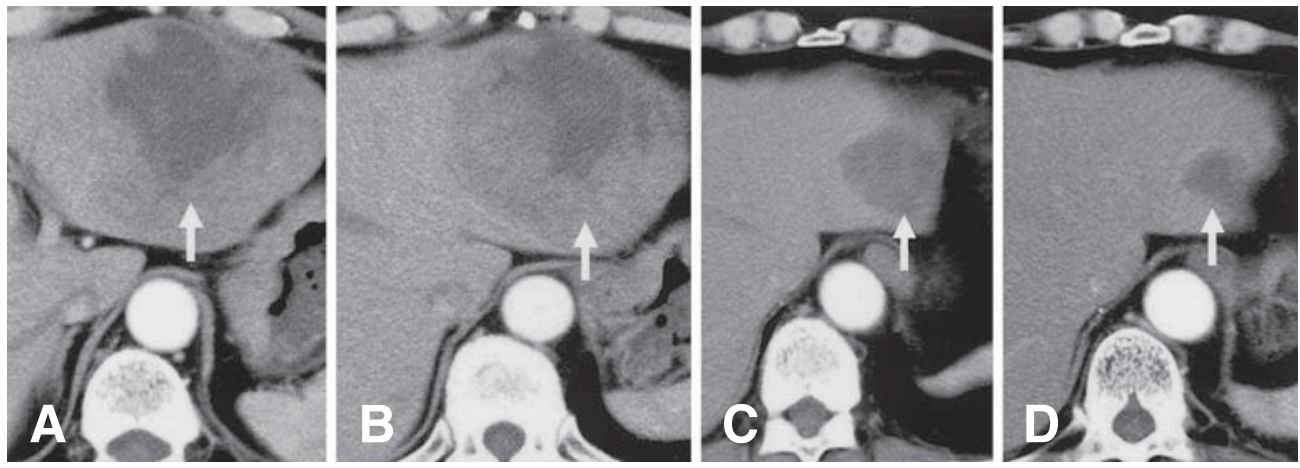

Fig. 2A-D. CT scans in case 2. A CT scan before chemotherapy was begun, showing a $94 \times 80$-mm metastatic tumor in the left lobe of the liver (arrow). B CT scan after two courses of S-1+ cisplatin (CDDP). The liver metastasis (arrow) was almost the same size as in the previous scan. C CT scan after 6 administrations of PTX. The liver metastasis (arrow) was reduced in size (38 mm; $59 \%$ reduction). D CT scan after 20 administrations of PTX. The liver metastasis (arrow) was further reduced in size
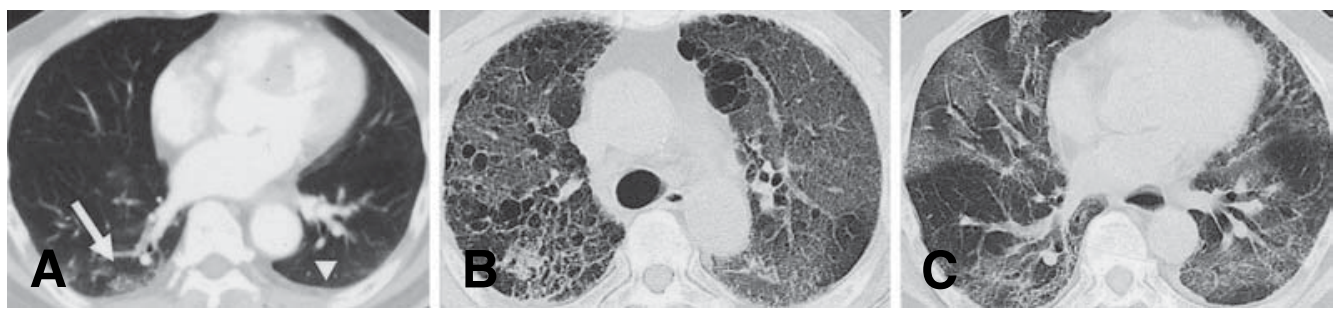

Fig. 3A-C. CT scans in case 2. A CT scan before PTX chemotherapy, showing slight consolidative changes (arrow) and subpleural lines (arrow head). B, C CT scans 2 days after 21 administration of PTX, showing extensive bilateral areas of groundglass attenuation with honeycomb changes

\section{Discussion}

The principal mechanism of action of PTX is the induction of stable polymerization of microtubules by the inhibition of microtubule disassembly. Because microtubules are essential for cell division, cells exposed to PTX are arrested in the premitotic G2 phase and fail to divide [1]. Three complications have been reported with PTX-induced pneumonitis: allergic reaction [3], type IV hypersensitive reaction [4], and cytotoxicity [5]. Stable polymerization of microtubules causes macrophages to release tumor necrosis factor (TNF)- $\alpha$ and interleukin (IL)-1, which have an antitumor effect [6]. However, these cytokines may have a cytotoxic effect on normal lung cells, and fibrosis may occur during the healing process [5]. This mechanism was suggested by an autopsy that showed marked fibrosis around shrunken metastatic tumors of the lung and liver [5]. The prevalence of grade 3 or more severe interstitial pneumonitis was shown to be approximately $0.73 \%$ (23/3169 cases) in a clinical trial in Japan that progressed until December 2002 [2]. Interstitial pneumonitis was more frequently reported with weekly or biweekly administration $(1.41 \%)$ than with tri-weekly administration
$(0.27 \%)$ [2]. We experienced 2 cases of pneumonitis among 210 patients treated with PTX at Kameda Medical Hospital and 62 patients treated with PTX at Misawa City Hospital, putting its frequency at approximately $0.73 \%(2 / 272)$. It was also reported that pneumonitis occurred more rapidly with weekly regimens than with tri-weekly regimens [7]. In post-marketing research in Japan, 20 cases of pneumonitis were reported in gastric cancer patients receiving PTX, with 13 recoveries [8]. The 7 patients who did not recover had been treated weekly with PTX monotherapy, receiving no combination therapy. No difference in the total dose of PTX was observed between those who died and those who recovered, so it seems that complications may develop at any time [8]. Some studies have reported that those undergoing combination therapies with taxanes and other agents, such as gemcitabine, had a higher risk of pneumonitis [9]. Similarly, pneumonitis was also reported in patients treated with docetaxel [10]. Our two patients had minimal consolidative changes in their lungs before treatment, and the presence of these charges may be one of the risk factors for pneumonitis. In addition, a long history of cigarette smoking may play a role in the development of PTX- 
induced pneumonitis, because smoking itself is recognized as one of the risk factors for interstitial pneumonitis or idiopathic pulmonary fibrosis [11]. It is important to consider symptoms such as cough and low-grade fever during treatment with PTX, as potential indicators of the possibility of onset of pneumonitis. PTX-induced pneumonitis needs to be considered in the differential diagnosis when patients treated with PTX present with respiratory symptoms. Infection must be ruled out, and a steroid should be administered during the early stages, because there are some case reports that early administration of steroids was effective for the treatment of drug-induced pneumonitis [12].

\section{References}

1. Donehower RC, Rowinsky EK. An overview of the experience with Taxol (paclitaxel) in the U.S.A. Cancer Treat Rev 1993; 19(Suppl):63-78.

2. Drug information (Paclitaxel) 7th revision. Tokyo: Bristol Myers Squibb; 2005.

3. Weiss RB, Donehower RC, Wiernik PH, Ohnuma T, Gralla RJ, Trump DL, et al. Hypersensitivity reactions from Taxol. J Clin Oncol 1990;8:1263-68.
4. Fujimori K, Yokoyama A, Kurita Y, Uno K, Saijo N. Paclitaxelinduced cell-mediated hypersensitivity pneumonitis. Diagnosis using leukocyte migration test, bronchoalveolar lavage and transbronchial lung biopsy. Oncology 1998;55:340-44.

5. Hasegawa K, Maruyama M, Ebuchui M. A case of paclitaxelinduced acute bilateral pneumonitis in advanced gastric cancer. Jpn J Cancer Clin 2005;51:447-53.

6. Bogdan C, Ding A. Taxol, a microtubule-stabilizing antineoplastic agent, induces expression of tumor necrosis factor alpha and interleukin-1 in macrophages. J Leukoc Biol 1992;52:119-21.

7. Taghian AG, Assaad SI, Niemierko A, Kuter I, Younger J, Schoenthaler R, et al. Risk of pneumonitis in breast cancer patients treated with radiation therapy and combination chemotherapy with paclitaxel. J Natl Cancer Inst 2001;93:1806-11.

8. Report of adverse events of paclitaxel. Tokyo: Bristol Myers Squibb; 2005.

9. Thomas AL, Cox G, Sharma RA, Steward WP, Shields F, Jeyapalan K. Gemcitabine and paclitaxel associated pneumonitis in non-small cell lung cancer: report of a phase I/II dose-escalating study. Eur J Cancer 2000;36:2229-34.

10. Read WL, Mortimer JE, Picus J. Severe interstitial pneumonitis associated with docetaxel administration. Cancer 2002;94: 847-53.

11. Baumgartner KB, Samet JM, Stidley CA, Colby TV, Waldron JA. 1. Cigarette smoking: a risk factor for idiopathic pulmonary fibrosis. Am J Respir Crit Care Med 1997;155:242-8.

12. Nomura M, Inoue Y, Fujita S, Sakao J, Hirota M, Souda S. A case of drug-induced interstitial pneumonitis after gemcitabine treatment for advanced pancreatic cancer. Gan To Kagaku Ryoho (Jpn J Cancer Chemother) 2006;33:123-7. 